\title{
SDT analysis of binary decisions and sensory intensity ratings to noxious thermal stimuli*
}

\author{
W. CRAWFORD CLARK $†$ and DONALD J. DILLON \\ New York State Psychiatric Institute \\ and Department of Psychiatry, Columbia University, New York, New York 10027
}

\begin{abstract}
During three sessions, each of 24 Ss responded to noxious thermal stimuli, using the following judgments: binary decision, S responded "high" or "low"; sensory intensity rating. S rated his sensory experience along a thermal intensity continuum; and concurrent report, S's binary decision was followed by an intensity rating. The binary-decision $\mathrm{d}^{\prime}$ was significantly higher than the rating $\mathrm{d}^{\prime}$, suggesting that $\mathrm{Ss}$ could not maintain multiple thermal criteria in a consistent fashion. The criteria for pain obtained with single and concurrent intensity rating judgments did not differ. These results suggest that the most efficacious and valid method for the study of experimental pain is to obtain concurrent responses, and to use binary decisions to compute $d$ ' and sensory intensity ratings to locate $S$ 's criterion for reporting pain.
\end{abstract}

SDT should prove to be particularly useful in the study of pain perception, since the response, "pain," clearly depends not only upon sensory variables such as stimulus intensity, but also upon the attitude of $S$ towards the test situation. For example, an anxious $S$ who fears injury may test his control over $E$ by responding. "pain," to relatively low-intensity stimuli; if $E$ obeys and ceases to raise the stimulus intensity, S's anxiety is reduced. The strong attitudinal component which is present in pain perception may explain the inconsistent thresholds which have been reported. For example. mean thermal pain thresholds obtained by different investigators vary between 182 and $348 \mathrm{mcal} / \mathrm{secXcm}{ }^{2}$ (Hardy, Wolff, \& Goodell, 1952) and individual thresholds range from 170 to $472 \mathrm{mcal} / \mathrm{secXcm}^{2}$ (Clausen \& King, 1950). The use of SDT is essential when groups differing in age, sex, socioeconomic status, etc., are being compared, since the threshold differences which are frequently reported are as likely to be caused by attitudinal differences as by sensory variation. For example, Clark and Mehl (1971) demonstrated that the raised threshold of older Ss was due to a higher response criterion for pain rather than to reduced sensory sensitivity. In an earlier study, Clark (1969) demonstrated the effect of instructional set on the pain threshold. The administration of a placebo as a potent analgesic sharply reduced the number of "pain" responses to thermal stimulation. Treatment of the data by the method of constant stimuli indicated that the pain threshold had been raised, that is, the placebo

*This research was supported by the National Institutes of Health through Grant RR-05650 from the General Research Support Branch and Grant NS-09263 from the National Institute of Neurological Diseases and Stroke.

†Requests for reprints should be sent to W. Crawford Clark. Department of Research Psychology, New York State Psychiatric Institute, 722 West 168 th Street, New York, New York 10032. mimicked the effects of an analgesic. However, analysis of the same data by SDT demonstrated that $d^{\prime}$ had not been altered. This indicates that the neurosensory input had not been attenuated. It was concluded that the sole effect of the placebo was to cause the $S$ to raise his response criterion for reporting pain. Clearly, the sensory decision procedure presents a much more complete and accurate description of the sensory and decision processes underlying $S$ 's behavior than do traditional psychophysical procedures.

Values of $d^{\prime}$ estimated by different procedures tend to be equal, and experimental evidence obtained in audition and vision usually substantiates this view (Egan, Schulman, \& Greenberg, 1959; Emmerich, 1968; Swets, Tanner, \& Birdsall, 1961). However, if the criterion fluctuates, as Wickelgren (1968) has suggested, then d's obtained by different procedures may be expected to vary. In particular, since it is probably more difficult to be consistent with respect to a large number of criteria than with a single criterion, rating d's may be expected to be lower than d's based on binary decisions. This may be especially true in pain experiments where $S$ must recognize perceptual qualities, or attributes, of the stimulus intead of simply detecting its presence or absence. In fact, there is some evidence in vision and audition (Markowitz \& Swets, 1967; Leshowitz, 1969) that $\mathrm{d}$ 's estimated by rating procedures may be lower, and perhaps more variable, than those obtained with the binary decision procedure.

The binary decision and sensory rating procedures possess both virtues and handicaps for pain research. The binary decision procedure is preferable if it is more sensitive, for such a procedure may be presumed to more accurately represent underlying sensory processes. Also, the binary decision procedure permits $E$ to reinforce right and wrong responses, and to compare S's criterion with that of an ideal observer calculated from the payoff matrix. However, the binary decision procedure does not reveal the complexity of the sensory experiences 
Table 1

Mean Discriminability (mcal/sec $\times \mathrm{cm}^{2}$ per $\mathrm{d}^{\prime}$ ) and Criterion Locus for the Binary Decision and Magnitude Rating Procedures

\begin{tabular}{lccccc}
\hline & \multicolumn{2}{c}{ Binary Decision } & & \multicolumn{2}{c}{ Magnitude Rating } \\
\cline { 2 - 3 } Response & $\begin{array}{c}\text { Discrimina- } \\
\text { bility }\end{array}$ & $\mathrm{L}_{\mathrm{X}}$ & & $\begin{array}{c}\text { Discrimina- } \\
\text { bility }\end{array}$ & $\mathrm{L}_{\mathrm{vfp}}$ \\
\hline Single & 24.24 & 1.25 & & 30.76 & 1.26 \\
Concurrent & 26.19 & 1.12 & & 35.67 & 1.26 \\
\hline
\end{tabular}

produced by noxious stimulation. The sensory intensity rating procedure permits determination of the criteria for reporting a wide variety of painful and nonpainful sensations (Clark, 1969). In addition, in a single session, the rating procedure yields an ROC curve from which estimates of the normality and of the relative variances of the underlying noise and signal-plus-noise distributions may be made.

The above considerations suggest that the most feasible approach would be to obtain two decisions following each stimulus presentation. Such a concurrent decision procedure would use the binary decision to yield a sensitive measure of $\mathrm{d}^{\prime}$, and the sensory rating decision to locate the criteria for reporting, "nothing," "hot," "pain," etc. However, it must be determined empirically whether the Ss can perform this more complex task without decreasing their sensory sensitivity or biasing their criterion for reporting pain.

The purpose of this study was to compare values of $d^{\prime}$ and pain criteria obtained by three SDT procedures: binary decision, sensory intensity rating, and concurrent judgment (in which the binary decision is followed by a magnitude rating response). A further purpose was to submit the data to statistical scrutiny; earlier studies had too few Ss to permit this.

\section{PROCEDURE}

The Hardy-Wolff-Goodell dolorimeter was used to present radiant heat stimuli of $3.0 \mathrm{sec}$ duration to two $3.5-\mathrm{cm}$-diam patches of India ink applied to either side of the midpoint between wrist and elbow on the volar surface of the nonpreferred forearm. The 100 stimuli were presented every $20 \mathrm{sec}$ to alternate patches. The approximate threshold of each S was obtained from responses to stimuli presented in a single ascending series of $100,150,200,225,250$, and, if necessary, 275 and $300 \mathrm{mcal} / \mathrm{secX} \mathrm{cm}^{2}$. On the basis of these responses, a particular set of stimuli, one relatively innocuous reference or "null" intensity and two stimulus Intensities at higher noxious levels, were tailored to each $\mathrm{S}$. The mean intensities (and approximate skin temperatures) were: reference intensity, $248 \mathrm{mcal} / \mathrm{secXcm}{ }^{2}\left(42.8^{\circ} \mathrm{C}\right)$; low-intensity stimulus, $269 \mathrm{mcal} / \mathrm{secXcm}^{2}\left(43.7^{\circ} \mathrm{C}\right)$, and high-intensity stimulus, $287 \mathrm{mcal} / \mathrm{secXcm}^{2}\left(44.7^{\circ} \mathrm{C}\right)$. The stimulus intensities were counterbalanced for order effects. Thus, in one-half of the session, 25 reference stimuli and 25 stimuli (either low or high) were presented in random order, followed $b y$ the reference and the remaining stimulus intensity. Each of 2.4 college students served in three sessions, counterbalanced for order effects. The three procedures differed only in the type and number of judgments required of the $\mathrm{O}$; the intensity of their stimuli, their number, and their random order of presentation remained constant throughout the experiment.

The Ss were informed of the a priori probability of occurrence of the stimuli $(0.5)$ and that, for the binary decision procedures, a hit would pay 2 cents and a false affirmative would cost 1 cent. According to SDT, this pay off matrix should cause $S$ to locate his criterion at $L_{x}=0.5$. Ss were paid at the end of the session to avoid the effects of knowledge of results on performance.

The three SDT procedures were: (a) Binary decision: The S judged whether the reference or a stimulus intensity had been presented by responding, "low" or "high." To make this decision, the $\mathrm{S}$ adopts a single criterion, $\mathrm{L}_{\mathbf{x}}$. (b) Sensory intensity rating: The $S$ evaluated his sensory experience using the following verbal rating scale: nothing - faint warmth - warm moderate - low heat - hot - very hot - very faint pain - pain - very painful - withdrawal. Thus, $S$ could utilize as many as 10 criteria, one between each of the 11 response categories on the rating scale. The "nothing," "faint warmth," and "withdrawal" categories were not used by any S. In the present study, the "very faint pain" criterion ( $\mathrm{L}_{\mathrm{vfp}}$ ), which separates painful from nonpainful sensory experiences was of particular interest. (c) Concurrent rosponse: During this session, the $\mathrm{S}$ made two responses following each stimulus presentation: a binary decision, followed by a sensory intensity rating. Thus, two d's and two criteria, $L_{x}$ and $L_{v f p}$, were computed.

\section{RESULTS AND DISCUSSION}

The data were treated according to methods outlined in Green and Swets (1966) and Clark (1969). Since somewhat different thermal intensities were used to stimulate each $\mathrm{S}$, a common measure was obtained by expressing sensory performance as $\mathrm{mcal} / \mathrm{sec} \mathrm{Xcm}^{2}$ per $\mathrm{d}^{\prime}$. This measure resembles a just noticeable difference with response bias removed; a low value reflects high sensitivity.

A 2 by 2 by 2 by 6 analysis of variance for repeated measures with Ss nested within order (Winer, 1962) was used to treat separately the measures of sensory performance, mcal/secX $\mathrm{cm}^{2}$, and the criteria $\mathrm{L}_{\mathrm{x}}$ and $\mathrm{L}_{\mathrm{vfp}}$. The variables were: number of responses, single vs concurrent; type of decision, binary vs rating; intensity of stimulus above the reference stimulus, low vs high; and order in which $S$ received the three methods. The mean values of $\mathrm{mcal} / \mathrm{secXcm}{ }^{2}$ per $\mathrm{d}^{\prime}$, averaged over intensity and order since they did not differ significantly, appear in Table 1. The most important finding was that single and concurrent responses yielded the same values of $\mathrm{mcal} / \mathrm{secXcm}{ }^{2}$ and criteria, $\mathrm{L}_{\mathbf{x}}$ and $\mathrm{L}_{\mathrm{vfp}}$. For both the single and concurrent decision procedures, the binary decision procedure proved to be more sensitive (lower value of $\mathrm{mcal} / \mathrm{secXcm}{ }^{2}$ per $\mathrm{d}^{\prime}$ ) than the rating procedure $(F=11.02, \mathrm{df}=1 / 18$, $\mathrm{p}<.01)$. Since an unstable criterion decreases $\mathrm{d}^{\prime}$ (Wickelgren, 1968), this suggests that Ss found it difficult to maintain a large number of thermal criteria in a consistent manner. Such a large difference between binary and rating decision procedures is seldom obtained with auditory and visual stimuli. Perhaps the thermal criteria cannot be retained in memory because there are relatively few linguistic labels for thermal, as compared to visual and auditory, stimuli.

The presentation of more than two stimulus 
intensities in a single session has been questioned on theoretical grounds. Luce (1963) points out that if more than two distributions exist along a common decision axis, then the decision axis is not a monotonic function of likelihood ratio. In the present experiment, where S judged but two stimulus intensities, the mean discriminability obtained with the sensory intensity procedure was $33.22 \mathrm{mcal} / \mathrm{sec} \mathrm{Xcm}^{2}$ per $\mathrm{d}^{\prime}$. This value is very close to that obtained in an earlier study (Clark, 1969), where $S$ discriminated among five stimulus intensities within a session. Apparently, Ss are as able to operate along a sensory-decision continuum extending through a large number of intensities, from zero to noxious, as they are when there are but two stimulus intensities to judge. This result suggests that Ss are basing their decisions directly on sensory intensity rather than on the likelihood ratio, or that Ss can make judgments even when a number of decision axes are embedded in Euclidian space.

Analysis of variance for location of the criteria revealed no significant differences between single and concurrent judgments (see Table 1). Thus, the number of decisions which followed a stimulus affected neither the low-high criterion of the binary decision procedure, $L_{x}$, nor the criterion for very faint pain, $L_{v f p}$ of the sensory estimation procedure. In order to maximize the expected value of the payoff matrix, an ideal $\mathrm{S}$ should have located his criterion at $L_{x}=.5$. However, the binary decision criteria for both single and concurrent decisions were significantly larger $(p<.001)$ in both instances according to the Wilcoxon matched pairs signed ranks test. Obviously, the Ss in this experiment did not behave as ideal observers, but were extremely conservative about reporting the presence of the higher intensity stimulus. Such excessive caution is frequently found (Swets, Tanner, \& Birdsall, 1961).

It may be concluded, since values of thermal discriminability and of the criterion for pain did not differ between the single and the concurrent judgment conditions, that the concurrent decision procedure is the most efficient means of studying the effects of noxious stimulation. When using this method, the binary response should be used to compare discriminability, expressed either as $\mathrm{mcal} / \mathrm{secX} \mathrm{cm}^{2}$ per $\mathrm{d}^{\prime}$ or as $\mathrm{d}^{\prime}$ directly, and the sensory rating response should be used to locate the criterion for pain, $\mathrm{L}_{\mathrm{vfp}}$.

\section{REFERENCES}

Clark, W. C. Sensory-decision theory analysis of the placebo effect on the criterion for pain and thermal sensitivity $\left(d^{\prime}\right)$. Journal of Abnormal Psychology, 1969, 74, 363-371.

Clark, W. C., \& Mehl, L. Thermal pain: A sensory decision theory analysis of the effect of age and sex on $d^{\prime}$, various response criteria, and $50 \%$ pain threshold. Journal of Abnormal Psychology, 1971, 78, 202-212.

Clausen, J., \& King, H. E. Determination of the pain threshold on untrained subjects. Journal of Psychology, 1950,-30, 299-306.

Egan, J. P., Schulman, A. I., \& Greenberg, G. Z. Operating characteristics determined by binary decisions and by ratings. Journal of the Acoustical Society of America, 1959, 31. 768-773.

Emmerich, D. S. ROCs obtained with two signal intensities presented in random order, and a comparison between yes-no and rating ROCs. Perception \& Psychophysics, 1968, 3, 35-40.

Green, D. M., \& Swets, J. A. Signal detection theory and psychophysics. New York: Wiley, 1966.

Hardy, D. J., Wolff, H. G., \& Goodell, H. Pain sensations and reactions. Baltimore: Williams \& Wilkins, 1952.

Leshowitz, B. Comparison of ROC curves from one and two interval rating scale procedures. Journal of the Acoustical Society of America, 1969, 46. 399-402.

Luce, R. D. Detection and recognition. In R. D. Luce, R. R. Bush, and E. Galanter (Eds.), Handbook of mathematical psychology. Vol. 1. New York: Wiley, 1963. Pp. 103-189.

Markowitz, J., \& Swets, J. A. Factors affecting the slope of empirical ROC curves: Comparison of binary and rating responses. Perception \& Psychophysics, 1967, 2, 91-100.

Swets, J. A., Tanner, W. P., \& Birdsall, J. G. Decision processes in perception. Psychological Review, 1961, 68, 301-340.

Wickelgren, W. A. Unidimensional strength theory and component analysis of noise in absolute and comparative judgments. Journal of Mathematical Psychology, 1968, 5, 102-122.

Winer, B. J. Statistical principles in experimental design. New York: McGraw-Hill, 1962.

(Received for publication June 26, 1972; revision received January 30,1973 .) 